\title{
Les professions interdites du droit de grève au Bénin et en France
}

\section{Bertin Millefort Quenum}

\section{(2) OpenEdition}

1 Journals

\section{Édition électronique}

URL : https://journals.openedition.org/rdctss/1980

DOI : $10.4000 /$ rdctss. 1980

ISSN : 2262-9815

Éditeur

Centre de droit comparé du travail et de la sécurité sociale

\section{Édition imprimée}

Date de publication : 1 avril 2018

Pagination : 46-57

ISSN : 2117-4350

\section{Référence électronique}

Bertin Millefort Quenum, «Les professions interdites du droit de grève au Bénin et en France », Revue de droit comparé du travail et de la sécurité sociale [En ligne], 1 | 2018, mis en ligne le 01 novembre 2021, consulté le 12 novembre 2021. URL : http://journals.openedition.org/rdctss/1980 ; DOI : https:// doi.org/10.4000/rdctss. 1980

\section{(c) (i) $\odot$}

Revue de droit comparé du travail et de la sécurité sociale est mise à disposition selon les termes de la Licence Creative Commons Attribution - Pas d'Utilisation Commerciale - Pas de Modification 4.0 International. 


\title{
LES PROFESSIONS INTERDITES DU DROIT DE GRÈVE AU BÉNIN ET EN FranCE
}

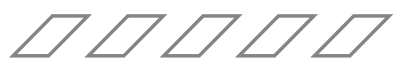

\section{RÉSUMÉ}

La consécration constitutionnelle du droit de grève en droit béninois et français ne rime pas nécessairement à une reconnaissance erga omnes à tous les travailleurs. Le législateur l'a interdit à certains travailleurs avec l'onction du juge constitutionnel. Au Bénin, comme en France, il est légalement limité pour certaines professions laissant apparaître convergence et spécificités nationales.

MOTS CLÉS: Droit constitutionnel, droit individuel, droit de grève, revendication, interdiction.

\begin{abstract}
Penal offense and then contractual fault, the strike became a constitutional right in France and Benin. Constitutional consecration of the right to strike in Benin and French law does not necessarily equate erga omnes recognition to all workers. The legislature banned some workers with the anointing of the constitutional judge. In Benin, as in France, it is legally limited for certain professions revealing convergence and national specificities.
\end{abstract}

KEYWORDS : Constitutional Law - Individual Right, Strike, Claim, Interdiction. 


\section{LES PROFESSIONS INTERDITES DU DROIT DE GRÈVE}

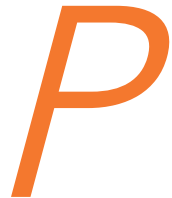

our certains auteurs, "la grève est un rapport de forces " ${ }^{1}$. La grève " est pour le juriste une sorte de paradoxe. Faire grève n'est-ce pas dresser contre l'État de droit existant ce que l'on oppose traditionnellement au droit : la force. Et pourtant, ceux qui font grève se réclament d'un droit, le droit de faire grève, le droit de grève $\aleph^{2}$. Le droit de grève est un droit de révolte. II ne serait pas un droit comme les autres droits. II renverrait, comme a pu l'écrire Jean-Marie Domenach, au « vide redoutable que faisait à Rome la plèbe en se retirant sur l'Aventin, une cassure dans la solidarité sociale, quelque chose de tragique et de religieux $»^{3}$.

En effet, « aux débuts et sauf exception, la grève a été un acte doublement illicite, au niveau pénal et au niveau civil. Il est évident que pour qu'un acte illicite devienne licite - à savoir conforme à l'ordre juridique - il faut que son illicéité soit supprimée sur tous les plans où elle existe ${ }^{4}$. Une telle affirmation est transposable en droit français en matière de grève puisque cette dernière est passée du statut d'infraction pénale ${ }^{5}$ à celui de droit consacré constitutionnellement. La dépénalisation de la grève à elle seule n'en a pas fait un droit. Il a fallu l'affirmation explicite des Constituants pour arriver à l'étape finale de la consécration du droit de grève par l'alinéa 7 du Préambule de la Constitution du 27 octobre 1946 selon lequel « le droit de grève s'exerce dans le cadre des lois qui le réglementent». Cette reconnaissance constitutionnelle a dépassé de loin son cadre intrinsèque, dans la mesure où cette liberté publique concédée aux travailleurs était censée déployer son ordonnancement juridique non seulement en France, mais aussi dans les territoires d'Outre-mer $»^{6}$. L'article 31 de la Constitution béninoise du 11 décembre 1990 proclame au titre des droits fondamentaux de la personne humaine que : «l'État reconnaît et garantit le droit de grève. Tout travailleur peut défendre, dans les conditions prévues par la loi, ses intérêts, soit individuellement, soit collectivement ou par l'action syndicale ».

En matière de jouissance du droit de grève, tous les travailleurs ne sont pas logés à la même enseigne. Certains sont interdits d'exercice du droit de grève. La jurisprudence du Conseil constitutionnel a validé cette prohibition. Pour le Conseil,

1 M. Bellanger, G. Darcy, "L'exercice du droit de grève au sein d'EDF », AJDA, 1997, p. 533.

2 P.D. Ollier, Droit du travail, éd. Armand Colin, 1972, p. 268.

3 Cité par P-D. Ollier, op. cit., p. 384.

4 N. Aliprantis, "La grève, un droit encore juridiquement méconnu», in Études dédiées à la mémoire de Pierre Orstscheidt, PUS, 2003, p. 15.

5 Il s'agit de la loi Le Chapelier des 14 et 17 juin 1791 qui a condamné les coalitions patronales ou ouvrières en prévoyant une amende de 500 livres et diverses incapacités civiques à l'encontre des grévistes contrevenants.

6 C.B. Amoussou, Droit béninois du Travail, Coll. Tête fertile, Star éditions, 2007, p. 7. 
«ces limitations peuvent aller jusqu'à l'interdiction du droit de grève aux agents dont la présence est indispensable pour assurer le fonctionnement des éléments du service dont l'interruption porterait atteinte aux besoins essentiels du pays ${ }^{7}$. C'est donc admettre qu' «il est des circonstances dans lesquelles la nature de la fonction exercée a paru commander la suppression pure et simple du droit de grève, et ceci de manière permanente, par voie législative $»^{8}$. Les personnes visées par cette interdiction législative sont en fait, certaines catégories d'agents publics qui, par leurs statuts particuliers, ont été privés de l'exercice du droit de grève. On constate une convergence sur ce point entre droit français et droit béninois (I) ainsi que des interdictions propres à chaque système juridique (II).

\section{I - CONVERGENCES ENTRE DROIT FRANÇAIS ET DROIT BÉNINOIS SUR L'INTERDICTION DU DROIT DE GRẺVE}

En raison des missions de sécurité publique ou de police qui leur sont confiées, certaines catégories de fonctionnaires sont privées par la loi du droit de grève. En droit français comme en droit béninois, les limitations à l'exercice du droit de grève ont été décidées à l'égard des militaires (A), des policiers (B) et des CRS (C) car « la discipline est la force principale des armées $"$ ?

\section{A - LES MILITAIRES}

Le Professeur Jacques Chevalier a énuméré les impératifs qui gouvernent le statut des militaires. Selon lui, «la spécificité militaire est d'abord organique et structurelle: le combat qui est la raison première du soldat implique une organisation particulière, monolithique, basée sur la hiérarchie, la discipline, l'unité, l'uniformité ${ }^{10}$. Le respect de ces impératifs explique les restrictions à l'exercice du droit de grève.

En France, c'est la loi du 13 juillet 1972 qui a consacré l'interdiction du droit de grève des militaires: "l'exercice du droit de grève est incompatible avec l'état militaire ${ }^{11}$. Certains ont pu justifier cette prohibition par le fait qu'une grève des militaires constituerait « une grave atteinte au principe de disponibilité permanente et surtout d'obéissance aux ordres reçus $»^{12}$. Le nouveau statut des militaires a reconduit in extenso la même formule lapidaire "l'exercice du droit de grève est incompatible avec l'état militaire ${ }^{13}$. À cette prohibition s'ajoute le fait que les militaires ne peuvent

7 Décision 79-105 DC du 25 juillet 1979, Rec., p. 33.

8 E. Devaux, La grève dans les services publics, tome I, PUF, 1995, 279-280.

9 R. (de) Bellescize, Les services publics constitutionnels, thèse, LGDJ, 2005, p. 413.

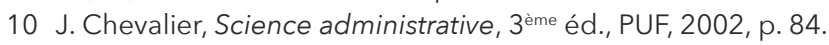

11 Cf. Article 11 de la loi $n^{\circ} 72-262$ du 13 juillet 1972 portant statut général des militaires, JORF du 14 juillet 1972, p. 7430.

12 M.-D. Charlier-Dagas, «Vers un droit syndical des personnels militaires?», RDP, 2003, p. 1073.

13 Cf. article L. 4121-4 alinéa 1er du Code de défense. 
pas mener une action, être affiliés à un syndicat, ou même adhérer à une association professionnelle ${ }^{14}$. Ainsi donc, l'exclusion du droit de grève s'accompagne de celle du droit syndical, la seule interdiction du droit de grève ayant paru insuffisante. C'est pourquoi, un autre auteur est allé jusqu'à soutenir que «le législateur peut, sans méconnaître la Constitution, interdire aux militaires le droit de grève, voire le droit de former des syndicats professionnels, notamment en raison d'une liaison de fait qui existe entre liberté syndicale et le droit de grève $»^{15}$. Malgré cette interdiction, des associations professionnelles ont été constituées par des militaires ${ }^{16}$. Mais par deux arrêts, le Conseil d'État ${ }^{17}$ a eu à rappeler que l'interdiction pour les militaires d'adhérer à des groupements professionnels est fondée historiquement sur le statut général des militaires ${ }^{18}$.

Toutefois, cette interdiction a été remise en cause par la Cour européenne des droits de l'homme. Cette juridiction a jugé que les restrictions, même significatives, qui peuvent être apportées aux modes d'action et d'expression d'une association professionnelle et des militaires qui y adhèrent ne doivent pas priver les militaires et leurs syndicats du droit général d'association pour la défense de leurs intérêts professionnels et moraux ${ }^{19}$. Selon le Professeur Antony Taillefait, cette jurisprudence prend «le contre-pied du Code de la défense, des décisions de l'autorité militaire et de la jurisprudence du Conseil d'État. Bref, si la liberté d'association des militaires peut faire l'objet de restrictions légitimes, l'interdiction pure et simple de constituer un syndicat ou d'y adhérer porte à l'essence même de cette liberté une atteinte prohibée par la Convention $»^{20}$. Un autre auteur y voit « la fin de l'interdiction absolue des syndicats au sein des armées $»^{21}$.

Au Bénin, aux termes de l'article de la loi n 81-014 du 10 octobre 1981 portant statut général des personnes militaires des forces armées populaires, « les personnels sont soumis en permanence aux règles suivantes: il leur est interdit de faire partie de groupements constitués pour soutenir des revendications d'ordre professionnel. Ils n'ont pas le droit de grève ". Il y a lieu de souligner qu'entre temps, lors du contrôle de constitutionalité du nouveau statut des forces armées, la Cour constitutionnelle dans l'une de ses décisions a considéré que « la Constitution ne prévoit aucune exception au

14 Cf. article L. 4121-4 alinéa 2 du Code de défense : « Lıexistence de groupements professionnels militaires à caractère syndical ainsi que lıadhésion des militaires en activité de service à des groupements professionnels sont incompatibles avec les règles de la discipline militaire ».

15 J. Robert, «Libertés publiques et défense », RDP, 1977, p. 951.

16 II s'agit respectivement de l'association nationale pour la défense de la parité des droits des administrés (ANDPDA) et de l'association de défense des droits des militaires (ADEFDROMIL).

17 CE 26 septembre 2007, M. Rémy, AJDA, 2007, p. 1846; CE 11 décembre 2008, Association de défense des droits des militaires, AJDA, 2008, p. 2369.

18 M. Ciavaldini, J. Millet, «Liberté d'expression collective des militaires : état du droit et développements jurisprudentiels récents », AJDA, 2009, p. 961.

19 CEDH 2 octobre 2014, n 10609/10 Matelly c/ France, AJDA, 2014, p. 1919.

20 A. Taillefait, « Militaires : restez regroupés», AJDA, 2014, p. 1969.

21 G. Poissonnier, "La fin de l'interdiction absolue des syndicats au sein des armées, " Dalloz, 2014, p. 2560. 
droit de grève pour telle ou telle catégorie. Le droit de grève proclamé et consacré par la Constitution du 11 décembre 1990 est un droit absolu au profit de l'ensemble des travailleurs dont les citoyens en uniformes des forces armées $»^{22}$.

Il est à déplorer que cette jurisprudence dans son interprétation très libérale et progressiste du droit de grève n'ait pas longtemps survécu. Elle a été remise en cause. En effet, l'interdiction d'exercice du droit de grève fut renouvelée par la loi du $1^{\text {er }}$ octobre 2011 portant interdiction du droit de grève des forces militaires et paramilitaires. D'abord, le législateur a essayé de préciser le contenu de la notion de personnels militaires qui comprend entre autres, les personnels de l'armée de terre ${ }^{23}$. Ensuite, aux termes de l'article 9 de ladite loi, il est précisé que « les personnels militaires sont tenus d'assurer leur mission en toute circonstance et ne peuvent exercer le droit de grève ». Enfin, la sanction attachée à la violation de la loi interdisant l'exercice du droit de grève est la radiation conformément aux dispositions de l'article 10 de la même loi.

En droit comparé et pour ne citer que la législation du Togo, l'article 8 du statut général des personnels militaires des forces armées prévoit que "les militaires jouissent de tous les droits et libertés reconnus aux citoyens. Toutefois, l'exercice de certains d'entre eux est soit interdit, soit restreint dans les conditions fixées par la présente loi » ${ }^{24}$. L'article 13 du même statut précise que "l'exercice du droit de grève est incompatible avec l'état militaire ». Cette disposition est la reprise exacte de la formule utilisée par le législateur français.

\section{B - LES POLICIERS}

En France, l'idée selon laquelle une interruption du service par le personnel de la police ne saurait être tolérée, a été formulée lors des débats parlementaires sur le statut des personnels de police avant d'être inscrite dans la loi. Selon le rapporteur de la loi, plus que tout autre corps de fonctionnaires, le corps des policiers est associé à la continuité inexorable de l'État ${ }^{25}$. Dans le même sens, il a été défendu qu' « il est impossible que vive un État si les hommes chargés d'assurer à tout moment sa sécurité peuvent refuser d'exécuter les ordres qui leur sont donnés. C'est pour nous, avant tout, une question d'autorité de la puissance publique $»^{26}$. On pourra aussi se référer à l'exposé des motifs de la loi portant statut général de la police. La police indique l'exposé des motifs, assume des responsabilités exceptionnelles. Sur elle repose la

22 Décision DCC 06-034 du 4 avril 2006, Président de la République, Recueil des décisions et avis de la Cour constitutionnelle, 2006, p. 157.

23 Cf. article $1^{\mathrm{er}}$ de la loi $\mathrm{n}^{\circ} 2011$-25 du $1^{\text {er }}$ octobre 2011 portant règles générales applicables aux personnels militaires, des forces de sécurité publique et assimilés en République du Bénin.

24 Cf. article 8 de la loi $n^{\circ} 2007-010$ du $1^{\text {er }}$ mars 2007 portant statut général des personnels militaires des forces armées togolaises, JORT du $1{ }^{\text {er }}$ mars 2007, p. 3.

25 D. Cordonnier, rapporteur DG, statut des personnels de police, JO débats AN, séance 20 septembre 1948, p. 6750.

26 J. Moch, "Statut des personnels de police », JO débats AN, séance du 20 septembre 1948, p. 6774. 
sûreté de l'État. Elle est gardienne de la loi, expression de la volonté de la Nation ${ }^{27}$. Dès lors, l'activité de la police ne peut connaître ni arrêt, ni grève. Or, pour montrer qu'il s'agit d'une interdiction absolue, la loi n'utilise pas le terme de grève ${ }^{28}$. En effet, l'article 2 al. 2 de la loi dispose que «toute cessation concertée du service, tout acte collectif d'indiscipline caractérisée pourra être sanctionné en dehors des garanties disciplinaires ${ }^{29}$. Même s'il n'a pas le droit de grève, le personnel de la police peut créer, adhérer ou se retirer librement d'un syndicat ${ }^{30}$. Il a même été créé des syndicats ne cachant pas leur affinité avec des partis politiques ${ }^{31}$.

Au Bénin, l'interdiction du droit de grève pour le personnel de la police se retrouve dans deux textes. La loi n 93-010 du 20 août 1997 portant statut des personnels de la_police nationale dispose en vertu de l'article 8 que «les personnels de la police nationale sont tenus d'assurer leur mission en toute circonstance. Ils ne peuvent exercer le droit de grève ". Cette interdiction a été réaffirmée par la loi du $1^{\text {er }}$ octobre 2011 portant interdiction du droit de grève des forces militaires et paramilitaires. Aux termes de l'article 9 de la même loi, « les personnels des forces de sécurité publique sont tenus d'assurer leur mission en toute circonstance et ne peuvent exercer le droit de grève ». Or, la loi a énoncé que "les personnels des forces de sécurité publique sont ceux de la police nationale ${ }^{32}$. À notre connaissance, le personnel de la police n'a jamais exercé le droit de grève.

En revanche, comme en droit français, les policiers béninois ont fini par obtenir le droit de créer ou d'appartenir à un syndicat. En effet, c'est la Cour constitutionnelle qui leur a reconnu le droit syndical suite à une longue et farouche opposition avec leur hiérarchie ${ }^{33}$.

27 Cf. Annexe $n^{\circ} 5353$, projet de loi relatif au statut des personnels de police, présenté au nom de M. André Marie, Président du Conseil des ministres, par Monsieur Jules Moch, Ministre de l'intérieur, doc. AN, $2^{\text {ème }}$ séance du 23 août 1948, p. 2043.

28 R. Bellescize de, op. cit., p. 397.

29 Cf. Article 2 alinéa 2 de la loi n 48-1504 du 28 septembre relative au statut spécial des personnels de police, JORF du 29 septembre 1948, p. 9532.

30 V. M. Bergès, Le syndicalisme policier en France (1880-1940), éd. L'Harmatan, col. Sécurité et société, 1995, 380 p; J.-L. Loubet del Bayle, «État du syndicalisme policier en France », Revue de l'administration publique, 1999, n 4, p. 434.

31 Syndicat Front national de la police, Fédération professionnelle indépendante de la police.

32 Cf. Article 1er de la loi nº 2011-25 du 1er octobre 2011 portant règles générales applicables aux personnels militaires, des forces de sécurité publique et assimilés en République du Bénin.

33 Décision DCC 05-059 du 7 juillet 2005, Recueil des décisions et avis de la Cour constitutionnelle, 2005 , p. 305. La reconnaissance du droit syndical a été affirmée au dernier considérant de la décision de la Cour : "Considérant qu'il ressort des éléments du dossier que d'une part, les textes régissant le personnel de la police nationale, notamment le Décret n 97-622 du 30 novembre 1997, reconnaissent au personnel de la police le droit de s'organiser en formation syndicale; que d'autre part, le Ministre de l'intérieur et de la sécurité et de la décentralisation ne rapporte pas la preuve de ce que la tenue du congrès constitutif du syndicat de la police serait de nature à porter atteinte à l'ordre public; qu'il y a lieu, par conséquent, de déclarer contraires aux articles précités de la Constitution la Lettre n²78/MISD/DGPN/SP-C du 13 avril 2004 du Directeur général de la police nationale portant suspension provisoire de la tenue du congrès constitutif du syndicat de la police ». 
Dans d'autres pays africains comme le Sénégal, le personnel de la police n'a ni le droit de grève, ni le droit syndical. En vertu de l'article 8 de la loi du 29 janvier 1978, "les membres des forces de police de tout grade, en activité de service ou en position de détachement ou de disponibilité, sont soumis en permanence aux règles suivantes : ils ne sont ni électeurs, ni éligibles. Ils ne jouissent ni du droit de grève, ni du droit syndical $»^{34}$.

\section{C - LES CRS}

En France, postérieurement à la reconnaissance du droit de grève, c'est une loi qui est venue réglementer ce droit au niveau des compagnies républicaines de sécurité. Elle prévoit (article 6) que pour les membres des compagnies républicaines de sécurité «toute cessation concertée ou non du service est assimilée à un abandon de poste et punie comme tel $\aleph^{35}$. En application de la loi, la jurisprudence a retenu que le ministre de l'intérieur n'a donc pas excédé de ses pouvoirs en prononçant une mesure de révocation à l'encontre d'un membre d'une compagnie républicaine de sécurité qui avait participé à un acte collectif d'indiscipline caractérisée et à une cessation concertée du service, sans l'avoir mis à même de demander la communication de son dossier ${ }^{36}$.

Au Bénin, les membres de la compagnie républicaine de sécurité font partie de la police nationale. Or, selon l'article 8 de la loi du 4 août 1993 portant statut des personnels de police nationale, "les personnels de la police nationale sont tenus d'assurer leur mission en toute circonstance. Ils ne peuvent exercer le droit de grève ». Cette interdiction du droit de grève fut renouvelée par l'article 9 de loi du $1^{\text {er }}$ octobre 2011 selon lequel «les personnels militaires ... sont tenus d'assurer leur mission en toute circonstance et ne peuvent exercer le droit de grève ». L'interdiction du droit de grève qui repose sur la nature exceptionnelle de l'activité qui ne saurait souffrir ni d'arrêt, voire de grève, est communément partagée par les droits béninois et français à l'égard des policiers et des gendarmes. D'autres prohibitions sont propres à chacun des deux systèmes.

34 Cf. Loi n 78-04 du 29 janvier 1978 Sénégal, in B. Diakhaté, "Le droit syndical au Sénégal », FES, 2005, p.45-46.

35 Cf. Loi n $n^{\circ}$ 47-2384 du 27 décembre 1947 portant réorganisation des compagnies républicaines de sécurité, JORF du 28 décembre 1947, p. 12494.

36 CE 10 mars 1972, Min. Intérieur c/ Santamaria, req. n 76065, Recueil Lebon, 1972, p. 207. 


\section{II - LES SPÉCIFICITÉS NATIONALES DE L'INTERDICTION DU DROIT DE GRÈVE}

Une rapide observation des catégories professionnelles interdites de grève révèle des cas de prohibition qui sont uniquement en vigueur en France $(A)$ alors que d'autres ne se rencontrent qu'en droit béninois (B).

\section{A- LA PHYSIONOMIE DES INTERDICTIONS SPÉCIFIQUES EN FRANCE}

En droit français, l'étude des statuts spéciaux révèle une interdiction du droit de grève pour les magistrats, les agents pénitentiaires et les préfets.

En ce qui concerne les magistrats, la terminologie utilisée est la suivante : « est également interdite toute action concertée de nature à arrêter ou entraver le fonctionnement des juridictions $\|^{37}$. $\|$ s'agit d'une sujétion imposée aux magistrats parce qu'un magistrat n'exécute pas un service comme un fonctionnaire, mais exerce une mission d'autorité, un pouvoir juridictionnel. II est évident que l'État ne saurait tolérer une paralysie de son action

juridictionnelle du fait même de ses détenteurs ${ }^{38}$. Malgré l'interdiction, des mouvements de grève sont parfois déclenchés par les magistrats. II en a été ainsi en 2011 de la « grève des audiences » déclenchée par les magistrats suite à la phrase jugée assassine du Président de la République à propos de l'affaire Tony Meihlon ${ }^{39}$.

En droit comparé, on retrouve également le retrait du droit de grève aux magistrats dans certaines législations africaines comme celle du Togo. Dans ce pays, l'article 9 du statut de la magistrature précise que «les revendications des magistrats sont portées devant le conseil supérieur de la magistrature [...] toute action concertée de nature à arrêter totalement le fonctionnement des juridictions est interdit » ${ }^{40}$. De même, au Sénégal, selon la loi, «les magistrats, même en position de détachement, n'ont pas le droit d'adhérer à un parti politique et toute manifestation leur est interdite. Ils ne peuvent ni se constituer en syndicat, ni exercer le droit de grève $»^{41}$. L'interdiction du droit de grève se retrouve dans les lois portant statut de la magistrature du Burkina-Faso (article 112) selon lequel « toute délibération politique est interdite au corps judiciaire. Toute manifestation d'hostilité au principe ou à la forme du gouvernement est interdite aux magistrats, de même que toute démonstration de nature politique incompatible

37 Cf. Article 10 de l'ordonnance ${ }^{\circ}$ 58-1270 du 22 décembre 1958 portant loi organique relative au statut de la magistrature, JORF du 23 décembre 1958, p. 11551.

38 Solon, Raison pour la justice, Dalloz, 1986, p. 173.

39 L. Priou-Alibert, "Rapports des services administratifs relatifs à l'affaire Tony Meilhon », Dalloz, 25 février 2011 (www.Dalloz.fr). Signalons pour mémoire, le mouvement de grève déclenché par le Syndicat de la Magistrature du 15 février 1978 à l'occasion de l'affaire Klaus Croissant, Solon, op. cit., p. 173.

40 Loi n $91-11$ du 21 août 1996 fixant statut des magistrats, JORT du 27 août 1996, nº 20 (bis), p. 6.

41 Cf. Article 10 de la Loi n 84-21 du 2 février 1984 portant statut des magistrats, in B. Diakhaté, «Le droit syndical au Sénégal », FES, 2005, p. 46. 
avec la réserve que leur imposent leurs fonctions $» .{ }^{42} \|$ en est ainsi également en Côte d'Ivoire ${ }^{43}$

En revanche, en droit béninois aucune disposition n'interdit aux magistrats le droit de grève ${ }^{44}$. Même le recours introduit par un Garde des sceaux devant la Cour constitutionnelle pour restreindre, voire interdire, ce droit suite à une grève déclenchée par les magistrats a été rejeté confirmant ainsi la légalité du droit de grève des magistrats ${ }^{45}$. Par la suite, la proposition de loi tendant à supprimer le droit de grève aux magistrats qui a fait l'objet d'un rejet au niveau de la Commission des lois ${ }^{46}$ a fini par être adopté le 28 décembre 2017. Mais la Cour Constitutionnelle dans une décision du 18 janvier 2018 vient à nouveau confirmer la non-conformité à la Constitution de la suppression du droit de grève des magistrats ${ }^{47}$.

42 Cf. loi organique n 050-2015/CNT du 25 août 2015 portant statut de la magistrature au Burkina-Faso.

43 Cf. article 16 de la loi 78/662 du 4 août 1972 portant statut de la magistrature en Côte d'Ivoire.

44 Aux termes de l'article 18, "Comme citoyens, les magistrats jouissent de la liberté d'expression, de croyance, d'association et de réunion. Ils sont libres de se constituer en association ou en toute autre organisation ou de s'y affilier pour défendre leurs intérêts, promouvoir leur formation professionnelle et protéger l'indépendance de la magistrature », Loi n 2001-35 du 21 février 2003 portant statut de la magistrature en République du Bénin, JORB du 15 mars 2004, p. 171.

45 Décision DCC 13-099 du 29 août 2013 (www.cour-constitutionnelle.org). En l'espèce, la ministre de la Justice avait déclaré : « Je gère des magistrats corrompus. Les magistrats de mon pays sont corrompus. La justice est totalement corrompue et la justice est par terre ». Cette déclaration abondamment relayée par la presse avait indigné les magistrats qui ont déclenché une série de grèves pour paralyser l'appareil judiciaire et ont exigé des excuses publiques de leur ministre de tutelle.

46 La modification envisagée est libellée ainsi (Art. 18 nouveau) : « Les fonctions judiciaires sont incompatibles avec tout mandat électoral politique. Toute délibération politique est interdite au corps judiciaire. Les magistrats sont inéligibles aux assemblées politiques. Les magistrats, même en position de détachement, n'ont pas le droit d'adhérer à un parti politique. Toute manifestation d'hostilité au principe ou à la forme du gouvernement de la République est interdite aux magistrats, de même que toute démonstration de nature politique incompatible avec la réserve que leur imposent leurs fonctions. Les magistrats ne peuvent se constituer en syndicat, ni exercer le droit de grève. Il leur est interdit d'entreprendre toute action concertée de nature à arrêter ou entraver le fonctionnement des juridictions ou d'y participer. Tout manquement par un magistrat aux dispositions du présent article est sanctionné par une mise à la retraite d'office ». La proposition de loi introduite par les députés de la mouvance a entraîné une forte mobilisation des syndicats de la magistrature, des centrales syndicales et des organisations de la société civile qui ont organisé plusieurs marches de protestation. Cf. entretiens avec certains magistrats et les secrétaires généraux de la CSA-Bénin, de la CGTB, Après la publication des noms des signataires dans toutes les juridictions du Bénin, certains députés signataires de la proposition de loi ont demandé le retrait de leur signature. Le rejet de la modification de la suppression du droit de grève aux magistrats au niveau de la Commission des lois a fait l'objet d'un recours en inconstitutionnalité. La décision de la Cour a retenu que la Présidente de la Commission des lois de l'Assemblée nationale n'a pas violé le règlement intérieur de l'Assemblée nationale. Cf. Décision DCC 14-179 du 22 septembre 2014 (www.cour-constitionnelle.org).

47 Cf. Décision DCC 18-001 du 18 janvier 2018, inédit. 
Quant aux agents pénitentiaires, ils sont soumis à une telle interdiction, puisque «toute cessation concertée du service, tout acte collectif d'indiscipline caractérisée de la part des personnels des services extérieurs de l'administration pénitentiaire est interdit. Ces faits, lorsqu'ils sont susceptibles de porter atteinte à l'ordre public, pourront être sanctionnés en dehors des garanties disciplinaires ${ }^{48}$. En ce sens, il a été jugé que la participation d'un représentant syndical à un mouvement collectif d'indiscipline ayant provoqué des dégradations de l'établissement pénitentiaire de Douai, portant ainsi atteinte à l'ordre public, justifiant une sanction disciplinaire sans consultation préalable du conseil de discipline ${ }^{49}$.

D'autres fonctionnaires sont privés du droit de grève. Il s'agit des fonctionnaires exerçant des fonctions d'autorité tels que les préfets et les sous-préfets qui n'ont ni le droit de grève, ni le droit syndical parce qu'ils sont considérés comme des " dépositaires de l'autorité de l'État $»^{50}$. Ils ont une obligation de loyauté vis-à-vis du gouvernement comme d'autres fonctionnaires tels que les recteurs

\section{B - LES EXCLUSIONS DU DROIT DE GRÈVE SPÉCIFIQUES AU BÉNIN}

Les interdictions spécifiques du droit de grève concernent les personnels de la douane et des eaux et forêts. Jusqu'à un passé récent, les douaniers pouvaient librement exercer le droit de grève. Ce n'est qu'à la faveur de la loi du 1er octobre 2011 que ce droit leur a été retiré. Pour y parvenir, le législateur, dans un premier temps, a assimilé les personnels de la douane aux forces de sécurité publique au niveau de l'article $1^{\text {er }}$ de ladite loi. Puis, il a clairement énoncé selon les dispositions de l'article 8 que «les personnels militaires, des forces de sécurité publique et assimilés sont tenus d'assurer leur mission en toute circonstance et ne peuvent exercer le droit de grève ». Cette réforme législative intervenue dans un contexte marqué par une série de grèves à la douane a fait l'objet de recours en inconstitutionnalité avant sa promulgation. En dépit des arguments évoqués par l'un des requérants ${ }^{51}$, la Cour a déclaré que l'interdiction du droit de grève des personnels de la douane est conforme à la Constitution ${ }^{52}$.

Pour justifier sa décision, elle a repris partiellement à son compte la position du Comité de la liberté syndicale lors de l'examen d'un conflit collectif au Nicaragua en $1994^{53}$. Le Comité a estimé donc que l'interdiction du droit de grève imposée aux travailleurs de ce secteur n'est pas contraire aux principes de la liberté syndicale, dès lors en particulier que les travailleurs en question bénéficient de garanties compensatoires

48 Cf. Article 3 de l'ordonnance n 58-696 du 6 août 1958, JORF du 7 août 1958, p. 7423.

49 TA Lille, 28 janvier 1997, M. D. Ferré, AFFP, 1997, n³ 3, p. 30.

50 Cf. Art. $1^{\text {er }}$ du Décret $n^{\circ}$ 2004-374 du 29 avril 2004 relatif aux pouvoirs des préfets, à l'organisation et à l'action des services de l'État dans les régions et départements JORF du 30 avril 2004.

51 Le personnel de la douane depuis le 18 juin 1990 ne fait partie ni des forces armées, ni des forces de sécurité et assimilés.

52 Décision DCC 11-065 du 30 septembre 2011, Annuaire béninois de justice constitutionnelle, Presse universitaire du Bénin, 2014, p. 571.

53 II s'agit du cas n 1719 où après une grève dans le secteur de la douane, il a été procédé au licenciement de 144 grévistes appartenant à l'Union Nationale des Employés

(www.ilo.org/dyn/normlex). 
de règlement des différends sous forme d'une participation aux négociations au sein de la commission de conciliation ${ }^{54}$. Mais par ailleurs, il a invité le gouvernement, afin de faciliter la reprise de relations professionnelles harmonieuses, à s'efforcer de favoriser la réintégration dans leur poste de travail des dirigeants syndicaux et des syndicalistes de l'Union Nationale des Employés (UNE) qui avaient été licenciés dans le secteur des douanes ${ }^{55}$. Dans ses observations sur la décision, le Professeur Alexis Essono-Ovono a estimé que « cette recherche de la légitimation de sa jurisprudence, en s'appuyant sur des normes internationales, est le propre des jeunes juridictions constitutionnelles recherchant l'acceptabilité sociales de leurs décisions $»^{56}$.

La suppression du droit de grève des douaniers béninois n'est pas une mesure unique en son genre. Dans certaines législations africaines telles que celle du Sénégal, le législateur depuis 1969 a prévu la même interdiction ${ }^{57}$. En revanche, en ce qui concerne le Niger, il y a lieu aujourd'hui de se réjouir que le syndicat national des agents des douanes (SNAD) a été rétabli dans ses droits au cours de l'année 2000 et exerce normalement ses activités désormais ${ }^{58}$.

Quant aux personnels des eaux et forêts, à l'instar des douaniers, ils sont aussi assimilés aux personnels des forces de sécurité publique. C'est donc l'article 8 de la loi du $1^{\text {er }}$ octobre 2011 précitée qui prévoit la même interdiction et en des termes identiques.

En droit comparé africain, le législateur sénégalais a prévu cette suppression du droit de grève aux personnels des eaux et forêts en 2005. Aux termes de l'article 8 de loi portant statut du personnel des eaux forêts et chasses, "le personnel des eaux, forêts et chasses de tous grades régi par le présent statut, en activité, en position de détachement ou de disponibilité est soumis aux règles suivantes: il n'est ni électeur, ni éligible; il ne jouit ni du droit de grève, ni du droit syndical $»^{59}$. Si au Bénin, aucune raison n'est évoquée pour justifier cette suppression, ce n'est pas le cas au Sénégal. Dans ce pays, la prohibition du droit de grève est expliquée dans l'exposé des motifs par la nécessité de renforcer la discipline, l'ordre et l'efficacité dans l'exercice des tâches de police forestières, de police de chasse, de lutte contre le braconnage, de lutte contre les feux de brousse et de participation aux comités de défense d'une part et d'autre part par l'harmonisation du statut du personnel des eaux, forêts et chasses avec l'uniforme militaire obligatoire et les attributs comparables à ceux des autres corps militaires de l'État ainsi que le port et l'usage d'armes à feu ${ }^{60}$.

54 Cf. Paragraphe $n^{\circ} 413$ du Cas $n^{\circ} 1719$, op. cit., (www.ilo.org/dyn/normlex).

55 Cf. Paragraphe $n^{\circ} 416$ c) du cas $n^{\circ} 1719$, op. cit., (www.ilo.org/dyn/normlex).

56 A. Essono-Ovono, "Obs. sous décision DCC 11-065 du 30 septembre 2011 », Annuaire béninois de justice constitutionnelle, Presses universitaires du Bénin, 2014, p. 585.

57 Cf. Article 8 de la loi n 69-64 du 30 octobre 1969 relative au statut du personnel de la douane au Sénégal.

58 Cf. Déclaration du secrétaire général du SNAD du 8 septembre 2000 cité par M. Oumarou, op. cit., p. 19.

59 Il s'agit de la loi n 2005-10 du 2005 portant statut du personnel des eaux forêts et chasses (www.jo.gouv.sn).

60 Cf. Exposé des motifs de la loi n $2005-10$ du 2005 portant statut du personnel des eaux forêts et chasses (www.jo.gouv.sn). 


\section{LES PROFESSIONS INTERDITES DU DROIT DE GRÈVE}

Délit pénal puis faute contractuelle, la grève est devenue un droit constitutionnel en France et au Bénin. Aux termes du Préambule de la Constitution française et de l'article 31 de la Constitution béninoise, c'est le législateur lui-même qui est invité à apporter les limitations qu'il jugerait nécessaires à l'exercice du droit de grève. Elles devraient résulter alors du cadre des lois qui le réglementent. La prohibition de l'exercice du droit de grève à certaines catégories professionnelles est communément partagée en droit français et béninois. Pour d'autres, l'interdiction est marquée par le sceau des spécificités nationales. Quant à l'éviction permanente du droit grève de certaines catégories professionnelles on constate une grande convergence entre en les droits béninois et français.

\section{BERTIN MILLEFORT QUENUM}

Directeur Général de la Fonction Publique par intérim.

Thèmes de recherche : Droit de grève, droit syndical, conventions internationales de l'OIT.

\section{Publications :}

B. Millefort Quenum, "Bénin », Actualités juridiques internationales, Revue de droit comparé du travail et de la sécurité sociale, $2013, \mathrm{n}^{\circ} 2$.

B. Millefort Quenum, "Bénin », Actualités juridiques internationales, Revue de droit comparé du travail et de la sécurité sociale, 2017, n³. 